\title{
Biodegradable microparticles for simultaneous detection of counterfeit and deteriorated edible products
}

Ivan Rehor, Sophie van Vreeswijk, Tina Vermonden, Wim E. Hennink, Willem K. Kegel, H. Burak Eral*

Dr. I. Rehor, S. van Vreeswijk, Prof. W. K. Kegel, Prof. H. B. Eral

Van't Hoff Laboratory for Physical and Colloid Chemistry, Debye Institute for Nanomaterials Science, Utrecht University, The Netherlands

E-mail: h.b.eral@tudelft.nl

Prof. H. B. Eral

Process \& Energy Laboratory, 3ME Faculty, TU Delft, The Netherlands

Prof. T. Vermonden, Prof. W. E. Hennink

Department of Pharmaceutics, Utrecht Institute for Pharmaceutical Sciences, Utrecht

University, The Netherlands

Keywords: Stop-Flow Lithography, Biodegradable, Sensor, Microparticle, Product safety

\section{Abstract}

In an era of globalized trade relations where food and pharmaceutical products cross borders

effortlessly, consumers face counterfeit and deteriorated products at elevated rates. This

manuscript presents multi-functional, biodegradable hydrogel microparticles that can provide

information on the authenticity and the potential deterioration of the tagged food or pharmaceutical formulations. These microparticles integrate spatially patterned authenticity code with two sensors - one detects possible presence of pathogenic microbes through monitoring $\mathrm{pH}$ while a second identifies products stored above optimal temperatures via optical monitoring of the microparticle degradation. Particles were synthesized from a biocompatible polymer and a photoinitiator, dextran modified with 2-

hydroxyethylmethacrylate (dex-HEMA) and riboflavin, respectively, using a continuous, high throughput method stop-flow lithography. The proposed synthesis approach also enables crosslinking with visible light bringing about additional flexibility to flow lithography. Model liquid and solid food and pharmaceutical products were successfully labeled with microparticles and the functionality of the sensors in aqueous solutions was demonstrated.

\section{Introduction}


Food and pharmaceutical products traded across the globe must fulfill strict quality criteria. However, potentially harmful products still appear on the market, raising serious public health concerns. Such products are either manufactured in substandard quality - a typical feature of counterfeit products ${ }^{[1-4]}$ - or undesired properties develop during inappropriate storage. Counterfeit consumer goods are on the rise globally. The situation is extraordinarily alarming in the case of pharmaceutical products, approximately $10 \%$ of all pharmaceuticals sold worldwide are counterfeits or of substandard quality, reaching $30 \%$ in developing countries $^{[1,2,5,6]}$. Equally alarming evidence can be found in food industry - in 2008 three hundred thousand babies were victimized along with 6 reported deaths due to substandard baby milk powder in China ${ }^{[7-9]}$. Use of authenticity labels on pharmaceuticals, such as special prints, holograms or other features, are a key part of the anti-counterfeit strategy recommended by World Health Organization $(\mathrm{WHO})^{[10]}$. Authenticity labels using isotopes ${ }^{[11]}$, chemicals $^{[12,13]}$, nanoparticles ${ }^{[14]}$ or microparticles ${ }^{[15]}$ were described in the literature and several solutions are already commercialized (DNATrax ${ }^{[16]}$, Truetag ${ }^{[17]}$ Taneeh $^{[18]}$ ). However the detection of these systems requires special instrumentation (dedicated spectrometer ${ }^{[17,18]}$ or polymerase chain reaction $\mathrm{kit}^{[16]}$, making verification by the end customer difficult. Microscale lithographic objects emerged as a logical alternative to nanoscale and molecular taggants as their size allows for nondestructive authentication using optical microscopic devices, which are easily accessible to the end customers thanks to rapid development of lowcost portable microscopic technologies ${ }^{[19]}$. Recent developments in micro manufacturing techniques allow for construction of highly elaborate micro objects that have the ability to carry the information about product i.e. serve as microsized barcodes. Numerous microbarcode designs are reported in literature and have been proposed for labeling pharmaceuticals $^{[17-19]}$, consumer goods ${ }^{[23,24]}$, or for multiplex assays ${ }^{[25]}$. The authenticity information is conventionally stored in the particle in the form of a code (binary ${ }^{[26]}$, color $^{[27-30]}$, barcode $^{[20,21]}, \mathrm{QR}$ code $\left.\mathrm{e}^{[22]}\right)$. The code is either imprinted in the barcode shape $\mathrm{e}^{[22,24,26]}$, its 
topography ${ }^{[24]}$ or, alternatively, the particle is divided into compartments of different compositions $^{[27,29]}$.

Generally, direct placement of the authenticity label onto the formulation provides superior security level over a label placed on the package, as it protects from gaining false authenticity through simple repackaging ${ }^{[31]}$. If an authenticity label is to be placed directly on or into the product, it must be constructed from biocompatible material to cause no harm to the consumer. Thus, it is preferred to design barcodes based on materials that are biodegradable and that have shown good biocompatibility characteristics in biomedical and pharmaceutical applications. The previously mentioned microbarcode designs, however, consist of nondegradable substances (either inorganic or polymeric) or even contain toxic substances, such as quantum dots ${ }^{[27]}$ or lanthanide salts ${ }^{[29,32,33]}$. To our best knowledge, the only biodegradable barcodes reported in literature are microfibers from polylactic-co-glycolic acid ${ }^{[20]}$ or alginate ${ }^{[34]}$. In both cases, one may speculate whether proposed fiber designs provide sufficient coding capacity needed for industrial scale manufacturing $\left(10^{10}\right.$ combinations is the current standard for batch identification in pharmaceutical).

Ensuring the degustation safety directly prior consumption is as important as verifying its authenticity. Pathogen contamination and growth inside a product is a major concern, especially with foods. In USA only, 48 million people get sick, 128000 are hospitalized and 3000 die from foodborne diseases every year ${ }^{[35]}$. The number of casualties worldwide reaches an alarming 420000 every year ${ }^{[36]}$. Even when not contaminated with pathogens, old or improperly stored product undergoes (partial) decomposition, which can alter its properties and, in certain cases, toxic effects arise. A notorious example from the pharmaceutical industry is the development of severe symptoms (nausea, vomiting, proteinuria, acidosis, glycosuria, and aminoaciduria) in patients treated with old, partly deteriorated tetracycline antibiotics $^{[37]}$. To protect a customer from consummation/use of contaminated or deteriorated products, every product bears an expiry date. This date, however, is relevant only when a 
product is stored under recommended conditions. As these conditions might have been violated in the supply chain, the consumer may avoid ingesting contaminated or deteriorated products, and prevent any associated risks, only by confirming the products' integrity directly prior to consumption. Although numerous technological solutions were proposed to check for example pathogen presence ${ }^{[38]}$ or product package integrity ${ }^{[39,40]}$, integrated commercial solutions are still missing on the market.

In this study, we report multifunctional hydrogel microparticles bearing a spatially patterned binary authenticity code capable of tagging $4.3 \times 10^{9}$ distinct products and two integrated sensors reporting the product safety. The ratiometric fluorescent $\mathrm{pH}$ sensor detects $\mathrm{pH}$ changes in tagged liquid products attributed to bacterial contamination - a common procedure in microbiology. The storage temperature sensor identifies products stored outside recommended storage temperature. This sensor can even detect short time ( $\sim$ several hours) exposure of the tagged product to elevated temperature and reports this information by permanent change of the particle shape. Owing to the biocompatible and biodegradable substrates used in the microfluidic synthesis, the microparticles can be placed directly on/into the edible products, providing orthogonal and more difficult to bypass security over current technologies placed on the package. We demonstrated that the developed microparticles are suitable for labeling solid and liquid formulations of model food and pharmaceutical products. The microparticles were visualized using a standard fluorescent microscope and a smartphone equipped with a low-cost lens attachment. The detection of microparticles with a common portable microscope enables rapid authenticity and product safety verification directly prior to consumption by consumers. We believe that the developed multifunctional microparticles may bring about a new integrated solution in addressing the global challenge of protecting customers from consuming counterfeit and deteriorated products.

\section{Results and Discussion}




\subsection{Particle Synthesis and Use as Authenticity Labels}

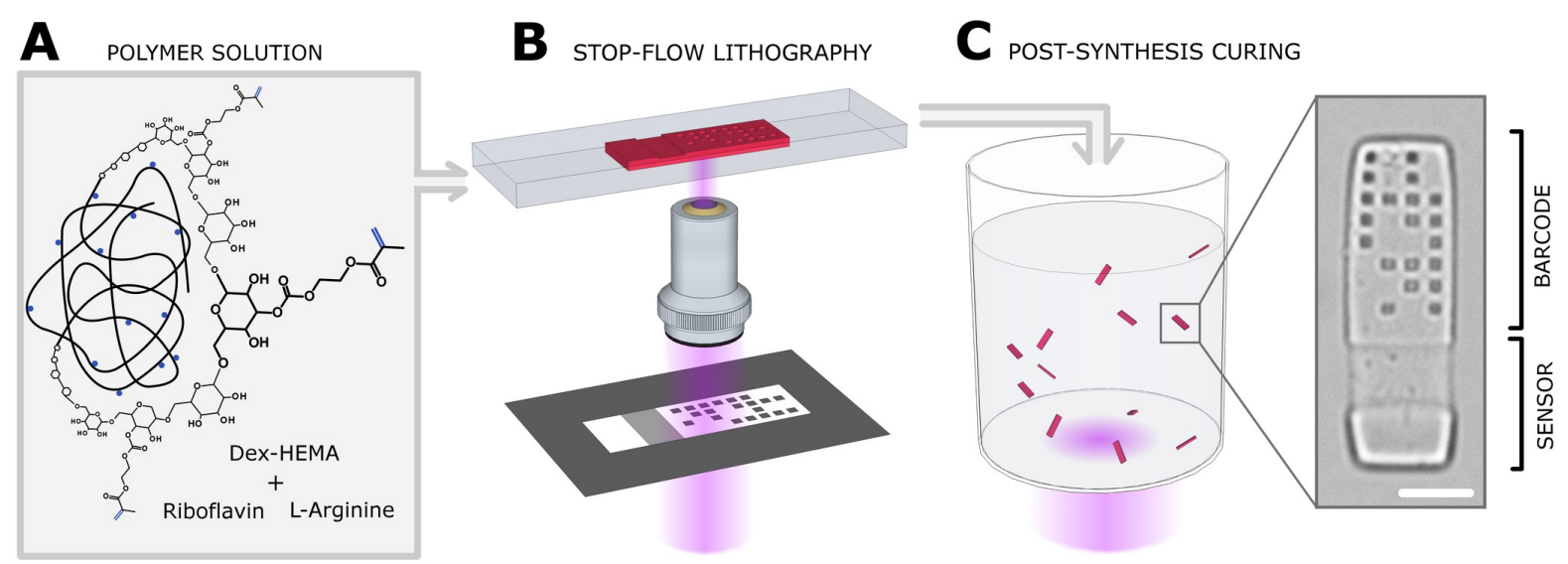

Figure 1. Scheme of the particle synthesis. Solution of biocompatible polymer (Dex-HEMA) and photoinitiator (riboflavin + L-arginine) in water (A) is pumped through a microfluidic channel and hydrogel microparticles are synthesized by light pulses through a mask placed at the field stop of the microscope (B). After particles are collected and diluted, they are irradiated again to increase their crosslinking density in a post-synthesis curing step. (C). The particle contains region with a binary code and sensor region used as a storage temperature sensor. Scale bar corresponds to $20 \mu \mathrm{m}$.

Stop-flow lithography was employed as a continuous technique for microparticle synthesis possessing significantly higher production rates compared to other lithographic methods ${ }^{[41]}$. The synthesis is described in detail in the experimental section and schematically depicted in Figure 1. In the previous works dealing with SFL, non-biodegradable polymers (mainly polyethyleneglycol-diacrylate - PEGDA) were used as substrates for lithographic hydrogel particle synthesis $^{[29,42]}$. An exception is the work of Hwang and coworkers ${ }^{[43]}$, in which hydrogel microparticles of simple geometries were synthesized from PEG bound to acrylates through hydrolyzable oligolactide linkers. Despite this change in chemical composition, the dominant mass fraction of the prepared hydrogels $(75 \%)$ was formed by the synthetic PEG polymer. It is general practice to use natural or naturally identical compounds in food and pharmaceutical products as much as possible to avoid possible undesired side effects of synthetic compounds. For this purpose, we utilized biocompatible Dex-HEMA polymer as a substrate for hydrogels ${ }^{[44]}$. Dex-HEMA consists of a natural polymer - dextran $(75 \%$ of total polymer mass) modified with 2-hydroxyethylmethacrylate moieties along the chain (Scheme 
in Figure 1-left). The connection between HEMA and dextran is achieved through hydrolytically labile carbonate esters, and therefore, hydrogels prepared by crosslinking DexHEMA degrade under biological conditions ${ }^{[45]}$. Dex-HEMA hydrogel particles were previously evaluated during clinical trials in human patients as a drug delivery carrier, exhibiting no harmful side effects ${ }^{[46]}$. Also products of Dex-HEMA hydrogels possess good biocompatibility ${ }^{[47]}$ - dextran is used as a plasma expander, and poly(2-hydroxyethyl methacrylate) is a polymer used in many biomedical products and other pharmaceutical applications $^{[44]}$. We have used riboflavin, vitamin B2, as a fully biocompatible photoinitiator $^{[48-50]}$ for crosslinking Dex-HEMA. Riboflavin (RF) requires an electron donor to work as a photoinitiator. L-arginine is a natural aminoacid which has been successfully implemented as an electron donor for riboflavin ${ }^{[50]}$. Using riboflavin and arginine allowed us to avoid traditional, possibly harmful ${ }^{[50,51]}$, phenone photoinitiators, which have been used up to now with SFL. Furthermore, riboflavin is photosensitive in the visible part of the spectrum ${ }^{[52]}$, hence we could use visible light (400-490 nm) for crosslinking. Avoiding UV exposure of the hydrogel is important for future applications of our system, such as production of cell laden hydrogels or hydrogels with biologically active, UV-sensitive molecules.

In order to produce mechanically robust particles with sufficiently long shelf life, we increased the particle crosslinking density by incorporating an additional post-synthesis curing step into the synthesis procedure (Figure 1C). After particles were flushed from the microfluidic channel, they were dispersed in a solution of riboflavin and arginine, and the whole suspension was irradiated again. In the post-synthesis irradiation, we nominally used a $365 \mathrm{~nm}$ UV lamp. To investigate if UV exposure can be avoided throughout the entire synthetic process, we also used a $405 \mathrm{~nm}$ diode laser instead of a UV lamp in several experiments (see experimental for more details). The choice of the light source in postsynthesis curing did not seem to have effect on the final stiffness or the decomposition behavior of microparticles: the 

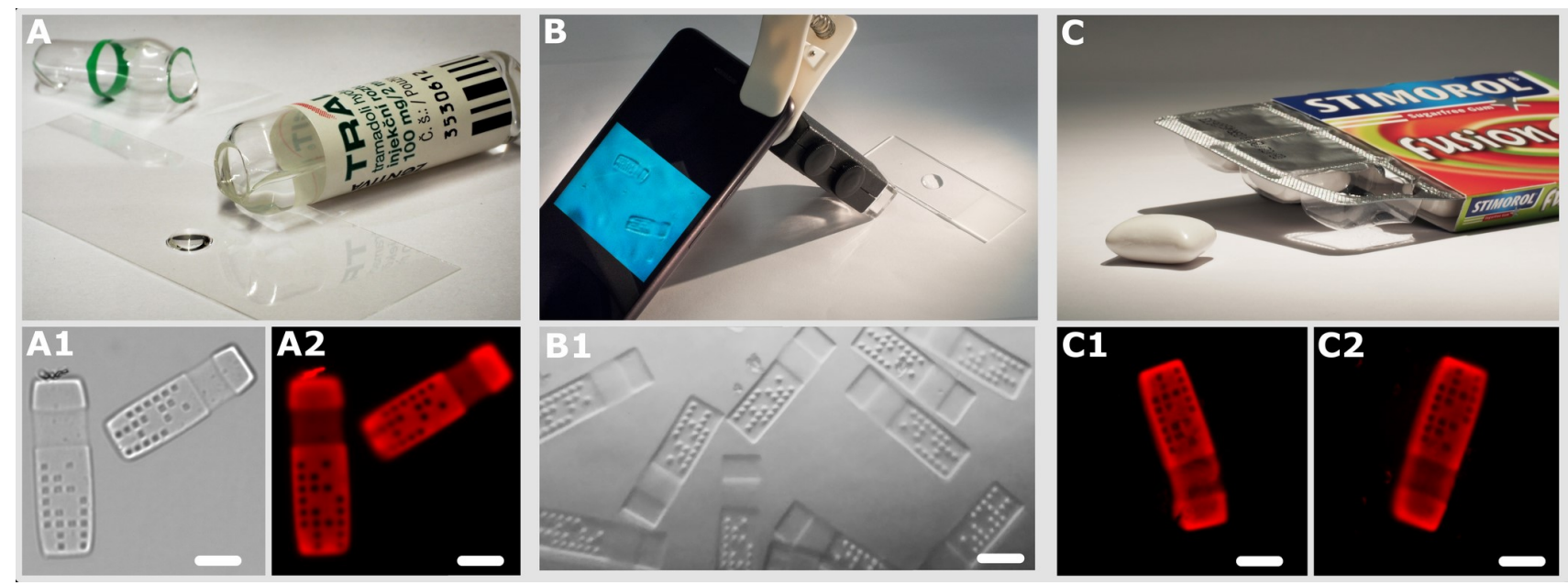

Figure 2. Model food and pharmaceutical products tagged with post-synthesis UV treated microparticles. A - labeled intravenous solution of Tramadol painkiller, transmission (A1) and fluorescence (A2) micrographs of microparticles dispersed inside the solution. B Particles in solution are visualized using a low-cost microscope attached to a smartphone (B1). $\mathrm{C}$ - labeled chewing gum and fluorescence microphotographs of particles on its surface $(\mathrm{C} 1$, $\mathrm{C} 2$ ). Scale bars correspond to $50 \mu \mathrm{m}$ in B1, and to $20 \mu \mathrm{m}$ in all other micrographs.

particles were stiff and no degradation was visible on aqueous solutions. However, more quantitative characterization is required in order to draw a final conclusion. The microscopy images of post-synthetically cured particles are shown in the Figures 1, 2A, 4. The microscopy image of microparticles synthesized without the post-synthesis curing step is provided in Figure S1.

The dimensions of synthesized particles were $100 \times 30 \times 25 \mu \mathrm{m}$ (standard deviation $>7 \%$ ), the first two dimensions are defined by the photomask, the third by the microfluidic channel height. The particles are large enough to be conveniently visualized using a standard laboratory microscope and, simultaneously, small enough to be completely invisible with a naked eye. The bright-field and fluorescence images (using incorporated rhodamine dye) of particles dispersed in solution are in Figure 2A. The particle contains binary code with $2^{32}$ $\left(\sim 4.3 \times 10^{9}\right)$ combinations. Similar coding system has been commercialized with SFLproduced microgels for biomolecule analysis ${ }^{[26]}$.To demonstrate the "on-the-spot" readability of microparticles prior to consumption by the end customers, we synthesized $2 \mathrm{x}$ larger particles $(200 \times 60 \times 25 \mu \mathrm{m})$ than our nominal size and visualized them using a cellphone 
camera with a microscopic lens attached (price $\sim 5$ EUR), as seen in Figure 2B. Thanks to the larger particle size, the code is readable despite the low resolution of the low-cost portable microscope.

The particles can be dried without losing their shape. Therefore, they could be used as labels for oral solid dose (OSD) formulations, i.e. tablets and capsules, which are by far the most common formulations of pharmaceuticals. Microparticles placed on OSD formulations only have anti-counterfeiting feature, as the sensors are only usable in liquid formulations. Fluorescence dye (acrylated rhodamine B) was covalently incorporated into the hydrogel to facilitate its reading with a fluorescent microscope ${ }^{[43]}$. The micrographs (Figure 2C) show that the particles keep their shape after drying, and the code remains readable on a model OSD surface (chewing gum).

\subsection{Sensing Functions of Particles in Aqueous Solutions}

Aqueous food and pharmaceutical products (drinks, pharmaceutical oral or intravenous solutions etc.) are in general more prone to a microbial contamination because microbes readily proliferate in aqueous solutions. The products dissolved in water also degrade faster than the dry forms of products. If the product is stored outside recommended conditions, such as elevated temperature, it can even deteriorate before the expiration date provided by the manufacturer. Therefore, we have incorporated two sensors into the microparticles that report on an actual condition of the labeled product. First, we focused on detection of a possible microbial contamination of a product. When an aqueous solution is contaminated with microbes, the solution $\mathrm{pH}$ drops ${ }^{[53]}$; thus, simply measuring the $\mathrm{pH}$ of a solution may indicate bacterial growth. This approach is routinely used in cell biology experiments, where cell growth media are labeled with phenol red, which changes its color from purple to orange upon decreasing $\mathrm{pH}$, thereby reporting bacterial contamination of cell culture. Ratiometric fluorescence $\mathrm{pH}$ detection is a powerful method with excellent signal-to-noise ratio which possesses reliable data even when measuring samples of complex composition due to the low 


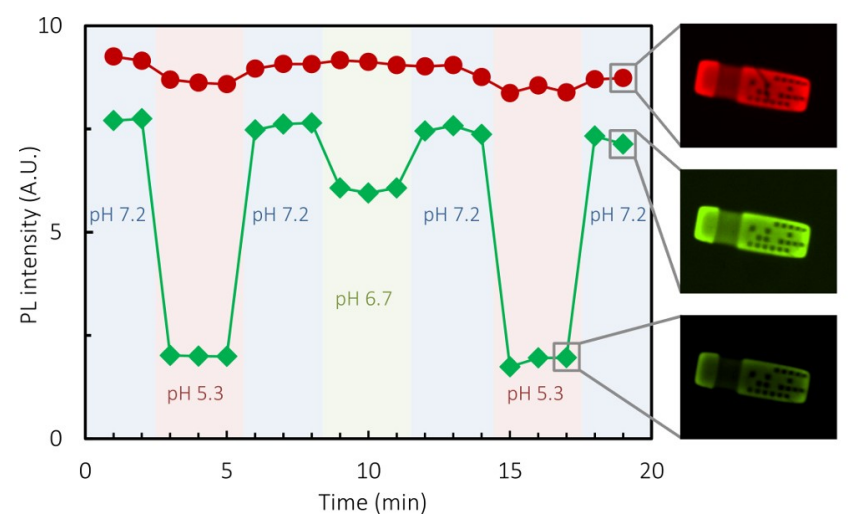

Figure 3. Plot of the particle's fluorescence response (green and red) to sudden $\mathrm{pH}$ changes. Emission intensities of both dyes of ratiometric sensor (fluorescein - responsive, rhodamine reference) are followed over time, while $\mathrm{pH}$ is changed every 3 minutes by buffer exchange.

probability of interference with measured sample ${ }^{[54]}$. Ratiometric $\mathrm{pH}$ detection has been used in various bioapplications such as measuring the $\mathrm{pH}$ in bacterial cultures ${ }^{[53]}$, inside tissues $^{[55]}$ or living cells ${ }^{[56]}$. To create ratiometric $\mathrm{pH}$ sensor, we covalently incorporated methacrylated fluorescein $(\mathrm{Fc})$ and rhodamine $\mathrm{B}(\mathrm{Rh})$ dyes into the hydrogel microparticles by adding them both into the pre-gel mixture of polymer and photoinitiator ${ }^{[43]}$. The emission intensity of the fluorescein dye is $\mathrm{pH}$ dependent hence it reports on a change of $\mathrm{pH}$. The emission peak of $\mathrm{Rh}$ at different wavelength is $\mathrm{pH}$ independent and serves as a reference. The fluorescence dyes were chosen with respect to the commercial availability of their acrylated analogues that allow simple covalent incorporation into the hydrogel. Furthermore Fluorescein and Rhodamine are easily detected by standard fluorescent microscopes, equipped with filter cubes that do not allow the freedom of choice in excitation and emission wavelength. We used low dye concentrations in the pregel mixture $(\sim 100 \mu \mathrm{M})$ to suppress Fluorescence Resonance Energy Transfer between the dyes ${ }^{[57]}$. These concentrations should be also sufficiently low to avoid any possible undesired side effects inside a body. We did an approximate estimation of the dye concentrations inside a tagged product to be below ppb (calculation can be found in Experimental section), which is deeply below legal toleration limits $(1.5 \mu \mathrm{g} \text { of undefined impurity in daily dose })^{[20,58]}$. The response of both dyes to changes in $\mathrm{pH}$ is plotted in the Figure 3. The plot shows quenching of the Fc emission when 
the $\mathrm{pH}$ drops, while the $\mathrm{Rh}$ emission remains constant. Fc emission response to the $\mathrm{pH}$ change is rapid (less than 1 minute) and its fluorescence intensity is fully recovered after subsequent $\mathrm{pH}$ increase. The ratio between $\mathrm{Fc}$ and $\mathrm{Rh}$ emissions is linearly proportional to the $\mathrm{pH}$ in the studied region from 6.0 to 7.5 (Figure S2) and the sensitivity is less than $0.5 \mathrm{pH}$ units. The presence of our sensor in a particle allows the observation of $\mathrm{pH}$ changes caused by pathogen growth and, thus, can prevent usage of a contaminated product by the consumers. We would like to point out, that a $\mathrm{pH}$ drop not always point to bacterial metabolical activity. Some pathogens do not lower the $\mathrm{pH}$ In fact; several changes in the product composition can alter the $\mathrm{pH}$. However many typical food contaminants (e.g. Escherichia coli) can be detected using simple sensor of this type ${ }^{[53]}$. Notably the $\mathrm{pH}$ check using our fluorescent sensor can be performed through a transparent package and therefore does not disturb the intactness of the product, unlike a conventional $\mathrm{pH}$ test.

Even when not contaminated by microbes, food and pharmaceutical products undergo aging and lose their original quality over long term storage. The storage conditions, in particular temperature is crucial in determining the deterioration rate of a product and, ultimately, the real expiration date. Therefore we developed a storage temperature sensor to identify products stored outside the suggested temperature range. The dex-HEMA polymer chemically degrades in aqueous solutions as the carbonate ester groups, connecting HEMA to dextran hydrolyze ${ }^{[44,45]}$. Due to the hydrolysis, polymer chains become more loosely connected to each other, which results in a swelling of a dex-HEMA hydrogel ${ }^{[45]}$ and subsequently leads to the disintegration of the entire particle. We decided to use the hydrogel swelling as a measure of its hydrolysis state. We have also developed an alternative methodology for this measurement, using the fluorescence decay of the particles during hydrolysis - more details can be found in Supplementary Information. Preliminary experiments of particle hydrolysis in water solution showed that the relative increase in overall particle dimensions (length and width) was only $20 \%$ during hydrogel hydrolysis before particles completely dissolved, 
which was not sufficient for precise determination of a hydrolysis state (plot in Figure 4A).

Therefore we designed our microparticles in the following way to increase the hydrogel response to the hydrolysis. The relative swelling of dex-HEMA hydrogels during hydrolysis decreases with their increasing initial crosslinking density ${ }^{[45]}$. The crosslinking density of our
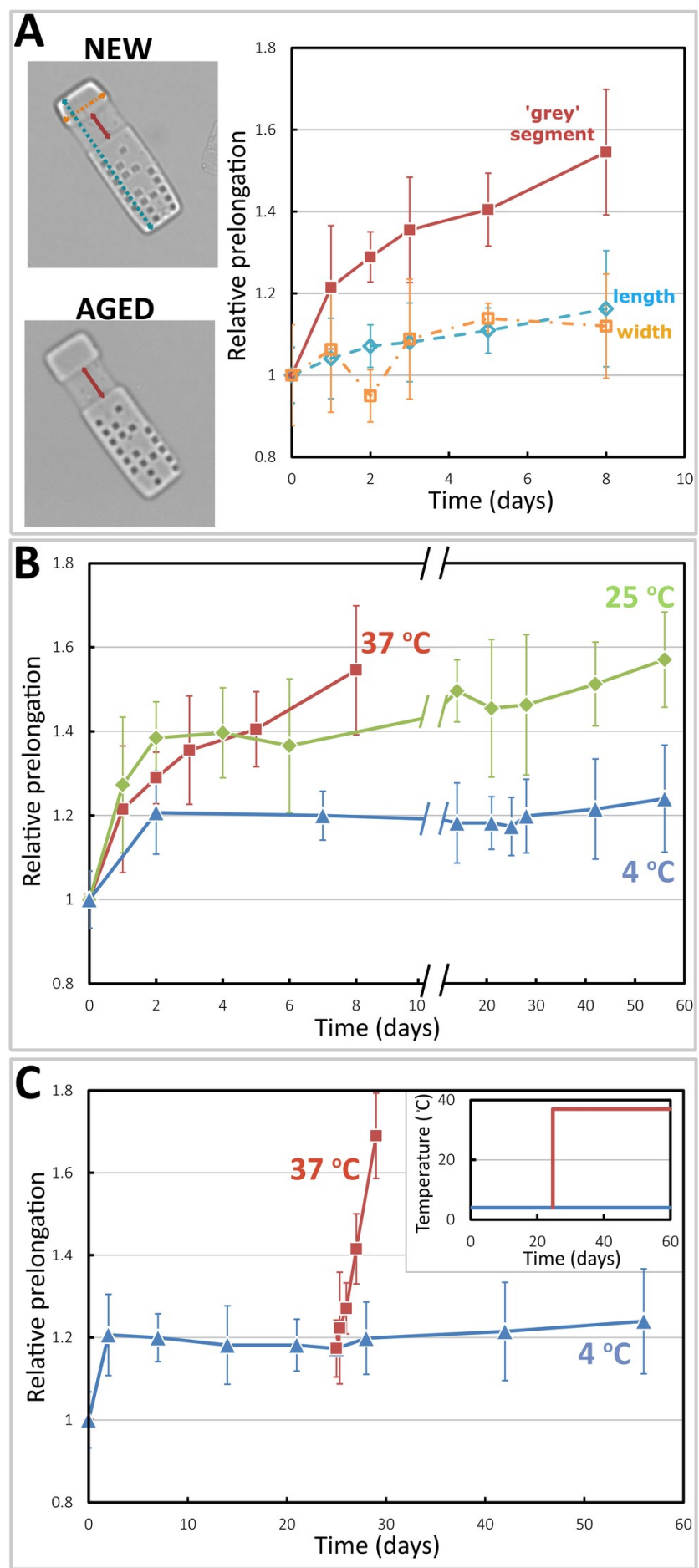

Figure 4. Tunable and temperature dependent swelling behavior of microparticles.

Microparticles stored in solution swell over time as the crosslinks in Dex-HEMA gel degrade (upper inset - prior to aging, lower - aged 8 days at $37^{\circ} \mathrm{C}$ ). The plot shows relative elongation along different directions in the microparticle over time. $\mathrm{B}-$ Relative prolongation 
of the sensory segment in particles stored at different temperatures $\left(4,25\right.$ and $37^{\circ} \mathrm{C}-$ triangles, diamonds and squares respectively). $\mathrm{C}$ - Relative swelling of 'grey' segment in particles stored at $4^{\circ} \mathrm{C}$ (triangles), compared to swelling in particles exposed to $37^{\circ} \mathrm{C}$ after 24 $\mathrm{d}$ at $4{ }^{\circ} \mathrm{C}$ (squares). Temperature profile is given in inset.

hydrogel particle is dependent (among other parameters) on the amount of light absorbed by the pre-gel solution during SFL synthesis. Thus we added a new segment into the photomask - a semitransparent stripe designed to transmit only $70 \%$ of light in order to decrease the irradiation intensity and subsequently the crosslinking density in the corresponding area of the particle - the 'grey' segment. Indeed in the performed ageing experiments the 'grey' segment in the particle exhibited a significantly steeper swelling curve, compared to the rest of the particle and reached $\sim 150 \%$ of its original length, before entire particle dissolved (Figure $4 \mathrm{~A}$ ). Notably the large error bars in the plots are due to the non-uniformity of the particles - each particle differs from each other in the crosslinking density and thus in the hydrolysis speed. We ascribe this non-uniformity to the poor control over experimental conditions during the post-synthesis curing step as omitting this step yields highly uniform particles. This issue is discussed in more detail in the Supplementary information. We are currently working on improving our experimental setup.

The figure 4B shows temperature dependency of the 'grey' segment swelling - the segment elongated faster when stored at elevated temperatures. The non-monotonic rates of the swelling in time are roughly similar to previously reported data on macroscopic Dex-HEMA hydrogels ${ }^{[45]}$. Next we focused on the response of the 'grey' segment to a sudden temperature change. We kept the particles at $4{ }^{\circ} \mathrm{C}$ for $\sim 3$ weeks, then divided the sample into two and heated up one half to $37^{\circ} \mathrm{C}$. The 'grey' segments of the particles exposed to the elevated temperature swelled rapidly (Figure 4C) and its elongation became significant (t-test) already after 24 hours exposure. In summary the sensor elongates faster when stored at elevated temperatures and even a short term exposure to an elevated temperature is reported by the sensor. The information is read simply by measuring the 'grey' segment length using a 
standard light microscope. This test can distinguish the product stored at recommended temperature, from a product exposed, even for a limited time, to an elevated temperature).

\section{Conclusion}

We developed a flow lithography process for manufacturing microparticles integrating authenticity and sensory functions, with non-harmful biodegradable chemicals. By careful choice of implemented chemistry, we avoided the commonly used UV irradiation in the lithographic process and replaced it with visible light. Synthesis of the microparticles with visible light brings about flexibility in experimental design (choice of microscope objectives, light source) and facilitates loading the hydrogels with a UV sensitive cargo, such as living cells or UV-cleavable drugs.

To demonstrate the feasibility of our method, we synthesized multifunctional biodegradable hydrogel microparticles as taggants for food and pharmaceutical products. The microparticles provide simultaneous information on the product authenticity and safety directly prior to consumption. The authenticity information is embedded in the microparticle topography as a binary code. The product safety of liquid products- the presence of the pathogens and the potential product deterioration due to improper storage - is reported through the sensors embedded in the microparticle structure. The authenticity code and the sensor information are acquired with a standard bright-field/fluorescent microscope (or even a cellphone in the case of authenticity). Incorporation of the sensors into the microparticle itself offers all-in-one solution with potentially broader applications than the previously proposed alternatives placed on the packages ${ }^{[59]}$.

The microparticle production is scalable, due to the continuous nature of stop-flow lithography so it can be implemented in industrial scale ${ }^{[26]}$. Simultaneously, it is a difficult technology to counterfeit, requiring advanced equipment and microfluidic skills. The natural degradation of the microparticles and the ability to directly place them on the products also 
limits possibilities for unauthorized manipulation, repackaging, reprocessing and dilution of original products, which is a common practice in counterfeiting. The proposed multifunctional system is orthogonal to other available methods therefore; it brings an additional layer of security, recommended in the WHO strategy to fight counterfeiting.

Our results demonstrate proof-of-concept verification of the product authenticity and condition "on-the-spot" directly prior to consumption. Thus, the multifunctional microparticles represent an integrated approach combining a microfluidic/lithographic technique that sculptures the microscale shape with molecular level chemical design enabling sensory and biodegradability functionality to protect consumers from potentially harmful counterfeit and deteriorated products.

\section{Experimental Section}

Materials. 1,1'-Carbonyldiimidazole (CDI),2-hydroxyethyl methacrylate (HEMA), 4methoxyphenol, Na2SO4, riboflavin, L-arginin, fluorescein O-methacrylamide, sodium azide, N,N-dimethylaminopyridin (DMAP) were purchased from Sigma Aldrich, methacryloxyethyl thiocarbamoyl Rhodamine B was purchased from Polysciences, SYLGARD® 184 silicone elastomer kit, used for production of polydimethylsiloxane (PDMS) channels was purchased from Dow Corning, dextran from Leuc. Spp., $\mathrm{Mr} \sim 40 \mathrm{kDa}$ (Sigma Aldrich) was dried over phosphorous pentoxide under vacuum at RT overnight, Molecular sieves ( $3 \AA$ Sigma Aldrich) were dried under vacuum at $250^{\circ} \mathrm{C}$ for 5 hours and used fresh. Dichloromethane, DMSO (HPLC, dried over sieves), reverse osmosis water was used in experiments (18.2 M $\Omega$ ). Synthesis of Dex-HEMA. HEMA- imidazoyl carbamate (HEMA-CI) was synthesized according to the previously published procedures ${ }^{[44]}$. The details can be found in the supplementary information. 2-Hydroxyethyl methacrylate modified dextran was synthesized according to previously published procedures ${ }^{[44]}$, with minor modifications. Briefly: Dextran ( $1 \mathrm{~g})$ was placed into a $40 \mathrm{~mL}$ flask together with $N, N$-dimethylaminopyridin (200 mg, 1.6 
mmol). DMSO was added $(9 \mathrm{~mL})$ and the flask was purged in 3 sequential steps of nitrogen and vacuum. Dextran and DMAP were dissolved under stirring (temperature was raised up to $70{ }^{\circ} \mathrm{C}$ to facilitate dissolution). HEMA-CI (430 $\mathrm{mg}$ of $90 \%$ product, $1.9 \mathrm{mmol}$ ) was added dropwise to the reaction mixture at $\mathrm{RT}$. The reaction was conducted at $\mathrm{RT}$ under the inert atmosphere for 3 days. Sodium phosphate buffer $(20 \mathrm{ml}, 0.1 \mathrm{M}, \mathrm{pH}=7.4)$ was added and the mixture was dialyzed against water for 4 days at $4{ }^{\circ} \mathrm{C}$ (the water was exchanged every 12 hours). The resulting solution was freeze-dried yielding $1.15 \mathrm{~g}$ of the product ( $86 \%)$. The obtained product was characterized using ${ }^{1} \mathrm{H}$ NMR. $\delta \mathrm{H}(400 \mathrm{MHz}, \mathrm{D} 2 \mathrm{O}) 6.02(\mathrm{bs}, 0.33 \mathrm{H}$, =CH'H' ${ }^{\prime}$ HEMA), 5.61(bs, 0.34H, =CH'H' HEMA), 4.85(bs, 1H, O-CH-O Dex $), 4.39(\mathrm{bs}$, 0.70H, N-CO-O-CH2 HEMA), 4.32(bs, 0.70H, C-CO-O-CH2 HEMA), 4.10-3.10 (m, 6H, Dex), 1.80(bs, 0.97H, -CH3 HEMA)

The number of HEMA groups per ribose unit of dextran (degree of substitution - DS) was calculated from a ${ }^{1} \mathrm{H}$ NMR spectrum, comparing integral intensity of six dextran protons multiplet between 3.2-4.1 ppm ( $\left.I_{\mathrm{DEX}}\right)$ to integral intensity of methylene group of HEMA at $1.75 \mathrm{ppm}\left(I_{\mathrm{HEMA}}\right)$ as stated in equation (1). The degree of substitution of Dex-HEMA used in the study was 0.35

$$
D S=\frac{2 \times I_{\text {HEMA }}}{I_{D E X}}
$$

Pre-gel nominal composition. $50 \mathrm{mg}$ of Dex-HEMA was dissolved in $180 \mu \mathrm{L}$ of the riboflavin saturated solution in water. After complete dissolution, L-arginine $(20 \mathrm{uL}$ of $5 \% \mathrm{w} / \mathrm{w}$ solution) was added. Then methacryloxyethyl thiocarbamoyl rhodamine B $(2 \mu \mathrm{L}, 12 \mathrm{mM}$ in DMSO) was added or/and fluorescein o-methacrylate stock solution ( $4 \mu \mathrm{L}, 62 \mathrm{mM}$ in DMSO) were added.

Microfluidic channel production. PDMS microfluidic channels were fabricated as previously described $^{[60]}$. First, the mixture of silicone elastomer base $(35 \mathrm{~g})$ and the curing agent $(5 \mathrm{~g})$ was poured over a silicon wafer with positive relief channels patterned with SU-8 photoresist 
(Microchem). The thickness of the PDMS layer was around $8 \mathrm{~mm}$. The wafer with PDMS was kept at $70{ }^{\circ} \mathrm{C}$ overnight then the PDMS layer was cut and peeled off. Next, a $1 \mathrm{~mm}$ hole was punctured through the PDMS on each end of the channel using biopsy needle. The channels were attached to glass slides with a thin layer of spincoated and semi-cured $\left(70{ }^{\circ} \mathrm{C}\right.$, 28 min, ratio base: curing agent $=10: 1 \mathrm{vol} / \mathrm{vol}$ ) PDMS layer. The whole device was then cured at $70{ }^{\circ} \mathrm{C}$ overnight. Ready channels of width $300 \mu \mathrm{m}$, height $30 \mu \mathrm{m}$ and length $2 \mathrm{~cm}$ were rinsed with ethanol MilliQ water and dried with a stream of nitrogen.

Microscope setup. Inverted microscope (Nikon Ti-E) was used for microparticle production and observation. A Hg lamp (Nikon Intensilight) was used as the light source for photocrosslinking $(<490 \mathrm{~nm}$ using dichroic mirror). The shutter of the source was controlled remotely. The photomasks were designed as a bitmap, transferred to diapositive slide, which was subsequently developed using wet photo process. A UV absorbing layer was present on the diapositive slide hence all light below $400 \mathrm{~nm}$ was blocked. For fluorescence microscopy cube filters for RITC and FITC (Semrock) were used.

Microparticle synthesis. The details of the SFL setup were described previously ${ }^{[42,61,62]}$. Compressed air was used to generate a pressure difference to induce flow in the channel. The input pressure was set to $0.5 \mathrm{psi}$ using a pressure gauge. The gauge outlet was connected to a 3 way solenoid valve which allows switching rapidly between atmospheric pressure (stop) and the input pressure (flow). The solenoid valve was controlled remotely. An inverted microscope was used for particle production. The PDMS channel was placed on the microscope stage. The $0.8 \mathrm{~mm}$ needle with flat end was filled with the pre-gel solution $(50 \mu \mathrm{L}$, standard composition - see above), inserted into the inlet hole and attached to the solenoid valve with a tube. A flat-end needle was inserted to the outlet hole and served for the collection of crosslinked particles. The solenoid valve and the Hg lamp shutter were programmed to cycle in a following way: I. PURGE: valve opened, shutter closed - 3 seconds; II. STOP: valve closed, shutter closed - 1 second; III. PRINT: valve closed, shutter 
open -1.2 seconds. Then the shutter was closed and simultaneously the valve was opened and the whole cycle was repeated. To avoid sticking of the synthesized particles to the channel walls, the irradiation position was changed by $150 \mu \mathrm{m}$ in the channel every 10 irradiations using the motorized stage. When the end of the channel was reached, the exposure spot was reset to the original position. After synthesis (typically several thousands of particles were prepared in one run), the channel was purged with MilliQ water $(500 \mu \mathrm{L})$ and particles were collected into an Eppendorf tube. For the post-synthetic curing step, $500 \mu \mathrm{L}$ of riboflavin saturated solution and $120 \mu \mathrm{L}$ of L-arginine solution $(5 \% \mathrm{w} / \mathrm{w})$ was added to the Eppendorf with collected particles. The Eppendorf was then placed under UV lamp (Blak-Ray® bench lamp, $365 \mathrm{~nm}, 40 \mathrm{~W}$ ) for 20 mins. The solutions carrying microparticles used for low-cost microscopy imaging (Figure 2b), were not irradiated with an UV-lamp, but with a $405 \mathrm{~nm}$ diode laser $\left(200 \mathrm{~mW}\right.$, spot size $\left.0.03 \mathrm{~cm}^{2}\right)$ for $20 \mathrm{~min}$. Then the particles were washed with water 4 times, allowed to sediment freely for 5 minutes between the washings.

Labeling pharmaceutical products. Tramadili hydrochloridum intravascular solution (Zentiva) was used as a model liquid pharmaceutical product. Approximately 50 particles dispersed in PBS $(50 \mu \mathrm{L})$ were pippeted into the solution. For a demonstration of the low-cost imaging, 50 particles of double size dispersed in PBS $(50 \mu \mathrm{L})$ were pippeted into the solution and visualized using Neewer 60X-100X Optical Zoom Mobile Phone LED microscope. Stimorol ${ }^{\circledR}$ Fusion Sugarfree gum was used as an oral solid dose (OSD) model. A droplet $(\sim 20$ $\mu \mathrm{L}$ ) of water dispersion containing particles was placed on the surface of the OSD and was dried by using a nitrogen stream. Particles were visualized on the surface using a wide-field fluorescence microscope (Nikon Ti-E, Hg Intensilight source) set to RITC channel. pH sensing. Particles were dispersed in $0.1 \mathrm{M}$ phosphate buffer of a $\mathrm{pH} 7.6$ and the particle images were acquired with a fluorescence microscope (Nikon Ti-E, Hg Intensilight source) on FITC and RITC channel. The buffer was replaced with phosphate buffer of $\mathrm{pH}, 7.0,6.5,6.0$ and 7.6 respectively. The interval between consecutive buffer replacements was 2 minutes, 
the microscope image was acquired every minute. The emission intensities were calculated from images (see image analysis section). The emission intensity of fluorescein was divided by the emission in intensity of rhodamine $\mathrm{B}$, to give a ratiometric value.

Estimation of a fluorescent dye content in a labelled product. We need no more than 100 microbarcodes to label $1 \mathrm{ml}$ of solution, the mass of one barcode particle is CA $75 \mathrm{pg}$ (estimated from dimenstions, density estimate $\sim 1.1 \mathrm{~g} / \mathrm{ml}$ ) which yields mass $/$ mass concentrations for barcodes in the range of several ppm. The dye concentration is $100 \mu \mathrm{M}$ in the hydrogel (this is the concentration in the pregel, after polymerization, and washing, it will further drop), which corresponds to roughly $50 \mu \mathrm{g}$ of the dye per $1 \mathrm{~g}$ of the hydrogel. It all together yields dye concentrations several orders of magnitude below $1 \mathrm{ppb}$ (mass/mass) inside a solution labelled with our microbarcodes). In the case of pharmaceutical industry an undefined impurity in the formulation is tolerated as long as it does not exceed $1.5 \mu \mathrm{m} / \mathrm{g}$ in daily dose. Expected amounts of the dye molecules are many orders of magnitude below this limit.

Particle hydrolysis experiments. Particles were stored at a given temperature in a PBS buffer with addition of $0.02 \% \mathrm{NaN} 3$ to inhibit bacterial growth and inspected periodically under the microscope in transmission mode using fluorescence microscopy (Nikon Ti-E, Hg Intensilight source) set to RITC channel.

Image analysis. The fluorescence intensity of a particle was determined by averaging the intensity along a line perpendicular to the main axis of the particle using Image J software. Background intensity was subtracted. The intensities were averaged over five particles.

superscripted and appear after the punctuation. ${ }^{[6]}$

\section{Supporting Information}




\section{Acknowledgements}

We acknowledge financial support from Utrecht University (Sustainability Talent Grant), from the European Union's Horizon 2020 research and innovation programme under the Marie Sklodowska-Curie grant agreement No. 705805) and Veni grant provided by Nederlandse Organisatie voor Wetenschappelijk Onderzoek (NWO) for H.B.Eral.

\section{References}

[1] “WHO | Substandard, spurious, falsely labelled, falsified and counterfeit (SSFFC) medical products," can be found under http://www.who.int/mediacentre/factsheets/fs275/en/, accessed: July, 2016.

[2] T. Kelesidis, I. Kelesidis, P. I. Rafailidis, M. E. Falagas, J. Antimicrob. Chemother. 2007, 60, 214.

[3] "Background Information - FIP - International Pharmaceutical Federation Counterfeit medicines," can be found under http://www.fip.org/www/index.php?page=menu_counterfeitmedicines_policy, accessed: December, 2016.

[4] "Poisoned medicine kills dozens of children in Nigeria - CNN.com," can be found under http://edition.cnn.com/2008/WORLD/africa/12/18/nigeria.poison.drugs/, accessed: September, 2016.

[5] "Ensuring the Safety and Integrity of the World's Drug, Vaccine, and Medicines Supply," can be found under http://www.cfr.org/pharmaceuticals-and-vaccines/ensuringsafety-integrity-worlds-drug-vaccine-medicines-supply/p28256, accessed: September, 2016. [6] V. N. Reports, "Black market malaria medication could cause dramatic rise in disease's spread," can be found under http://vaccinenewsdaily.com/stories/510532765-blackmarket-malaria-medication-could-cause-dramatic-rise-in-disease-s-spread, accessed: September, 2016. 
[7] Y. Cheng, Y. Dong, J. Wu, X. Yang, H. Bai, H. Zheng, D. Ren, Y. Zou, M. Li, J. Food Compos. Anal. 2010, 23, 199.

[8] J. M. in Beijing, Times Lond. 2008.

[9] "Ministry: 6 infants possibly died of tainted milk powder_English_Xinhua," can be found under http://news.xinhuanet.com/english/2008-12/01/content_10441344.htm, accessed: September, 2016.

[10] "WHO | IMPACT - The handbook," can be found under http://apps.who.int/impact/resources/handobook/en/index.html, accessed: September, 2016.

[11] L. A. Felton, P. P. Shah, Z. Sharp, V. Atudorei, G. S. Timmins, Drug Dev. Ind. Pharm. 2011, 37, 88 .

[12] D. Bansal, S. Malla, K. Gudala, P. Tiwari, Sci. Pharm. 2013, 81, 1.

[13] "Edible Barcodes for Food Safety \& Traceability SafeTraces," can be found under http://www.safetraces.com/, accessed: September, 2016.

[14] M. Puddu, D. Paunescu, W. J. Stark, R. N. Grass, ACS Nano 2014, 8, 2677.

[15] S. O. Meade, M. S. Yoon, K. H. Ahn, M. J. Sailor, Adv. Mater. 2004, 16, 1811.

[16] DNA Based Bar Code for Improved Food Traceability, accessed: September, 2016.

[17] “TruTag Technologies - Product Authentication \& Brand Protection," can be found under http://www.trutags.com/, 2015.

[18] “Taaneh: Authentication Technology, Anti Counterfeiting, Brand Protection Solutions," can be found under http://taaneh.com/, accessed: September, 2016.

[19] J. S. Cybulski, J. Clements, M. Prakash, PLOS ONE 2014, 9, e98781.

[20] C. Huang, B. Lucas, C. Vervaet, K. Braeckmans, S. Van Calenbergh, I. Karalic, M. Vandewoestyne, D. Deforce, J. Demeester, S. C. De Smedt, Adv. Mater. 2010, 22, 2657.

[21] K. Braeckmans, S. C. De Smedt, C. Roelant, M. Leblans, R. Pauwels, J. Demeester, Nat. Mater. 2003, 2, 169. 
[22] S. Han, H. J. Bae, J. Kim, S. Shin, S.-E. Choi, S. H. Lee, S. Kwon, W. Park, $A d v$.

Mater. 2012, 24, 5924.

[23] D. Dendukuri, D. C. Pregibon, J. Collins, T. A. Hatton, P. S. Doyle, Nat. Mater. 2006, 5,365 .

[24] H. J. Bae, S. Bae, C. Park, S. Han, J. Kim, L. N. Kim, K. Kim, S.-H. Song, W. Park, S. Kwon, Adv. Mater. 2015, 27, 2083.

[25] Y. Zhao, Y. Cheng, L. Shang, J. Wang, Z. Xie, Z. Gu, Small 2015, 11, 151.

[26] D. C. Pregibon, M. Toner, P. S. Doyle, Science 2007, 315, 1393;

http://www.fireflybio.com/, accessed: May, 2017.

[27] Y. Zhao, H. C. Shum, H. Chen, L. L. A. Adams, Z. Gu, D. A. Weitz, J. Am. Chem. Soc. 2011, 133, 8790 .

[28] H. Lee, J. Kim, H. Kim, J. Kim, S. Kwon, Nat. Mater. 2010, 9, 745.

[29] J. Lee, P. W. Bisso, R. L. Srinivas, J. J. Kim, A. J. Swiston, P. S. Doyle, Nat. Mater. 2014, 13, 524 .

[30] Y. Zhao, Z. Xie, H. Gu, L. Jin, X. Zhao, B. Wang, Z. Gu, NPG Asia Mater. 2012, 4, e25.

[31] D. deKeiffer, Am. J. Law Med. 2006, 32, 325.

[32] M. You, J. Zhong, Y. Hong, Z. Duan, M. Lin, F. Xu, Nanoscale 2015, 7, 4423.

[33] M. You, M. Lin, S. Wang, X. Wang, G. Zhang, Y. Hong, Y. Dong, G. Jin, F. Xu, Nanoscale 2016, 8, 10096.

[34] E. Kang, G. S. Jeong, Y. Y. Choi, K. H. Lee, A. Khademhosseini, S.-H. Lee, Nat. Mater. 2011, 10, 877.

[35] "Estimates of Foodborne Illness in the United States | Estimates of Foodborne Illness | CDC," can be found under https://www.cdc.gov/foodborneburden/, accessed: September, 2016. 
[36] World Health Organization, Foodborne Disease Burden Epidemiology Reference Group, WHO Estimates of the Global Burden of Foodborne Diseases, 2015.

[37] Frimpter GW, Timpanelli AE, Eisenmenger WJ, Stein HS, Ehrlich LI, JAMA 1963, $184,111$.

[38] M. S. Thakur, K. V. Ragavan, J. Food Sci. Technol. 2012, 50, 625.

[39] M. Smolander, E. Hurme, R. Ahvenainen, Trends Food Sci. Technol. 1997, 8, 101.

[40] X. Wang, H. Chen, Y. Zhao, X. Chen, X. Wang, X. Chen, TrAC Trends Anal. Chem. 2010, 29, 319.

[41] M. E. Helgeson, S. C. Chapin, P. S. Doyle, Curr. Opin. Colloid Interface Sci. 2011, 16, 106.

[42] D. Dendukuri, S. S. Gu, D. C. Pregibon, T. A. Hatton, P. S. Doyle, Lab. Chip 2007, 7, 818.

[43] D. K. Hwang, J. Oakey, M. Toner, J. A. Arthur, K. S. Anseth, S. Lee, A. Zeiger, K. J. Van Vliet, P. S. Doyle, J. Am. Chem. Soc. 2009, 131, 4499.

[44] W. N. E. van Dijk-Wolthuls, S. K. Y. Tsang, J. J. Kettenes-van den BoschW.E. Hennink, Polymer 1997, 38, 6235.

[45] W. N. E. van Dijk-Wolthuis, J. A. M. Hoogeboom, M. J. Van Steenbergen, S. K. Y. Tsang, W. E. Hennink, Macromolecules 1997, 30, 4639.

[46] K. D. F. Vlugt-Wensink, R. de Vrueh, M. G. Gresnigt, C. M. Hoogerbrugge, S. C. van Buul-Offers, L. G. J. de Leede, L. G. W. Sterkman, D. J. A. Crommelin, W. E. Hennink, R. Verrijk, Pharm. Res. 2007, 24, 2239.

[47] J. A. Cadée, L. A. Brouwer, W. den Otter, W. E. Hennink, M. J. A. van Luyn, J. Biomed. Mater. Res. 2001, 56, 600.

[48] B. Orellana, A. M. Rufs, M. V. Encinas, C. M. Previtali, S. Bertolotti, Macromolecules 1999, 32, 6570. 
[49] M. V. Encinas, A. M. Rufs, S. Bertolotti, C. M. Previtali, Macromolecules 2001, 34, 2845.

[50] S.-H. Kim, C.-C. Chu, J. Biomed. Mater. Res. B Appl. Biomater. 2009, 91B, 390.

[51] L.-T. Ng, S. Swami, C. Gordon-Thomson, Radiat. Phys. Chem. 2006, 75, 604.

[52] S. Kim, C.-C. Chu, Fibers Polym. 2009, 10, 14.

[53] X. Wang, R. J. Meier, O. S. Wolfbeis, Angew. Chem. 2013, 125, 424.

[54] M. H. Lee, J. S. Kim, J. L. Sessler, Chem Soc Rev 2015, 44, 4185.

[55] S. Schreml, R. J. Meier, O. S. Wolfbeis, M. Landthaler, R.-M. Szeimies, P. Babilas, Proc. Natl. Acad. Sci. 2011, 108, 2432.

[56] S. Bassnett, L. Reinisch, D. C. Beebe, Am. J. Physiol. - Cell Physiol. 1990, 258, C171.

[57] K. E. Sapsford, L. Berti, I. L. Medintz, Angew. Chem. Int. Ed. 2006, 45, 4562.

[58] D. J. Snodin, Organic Process Research \& Development 2010, 14, 960.

[59] “Temperature Monitoring \& Temperature Sensitive Labels," can be found under http://timestrip.com/temperature-monitoring/, 2014.

[60] H. Z. An, E. R. Safai, H. Burak Eral, P. S. Doyle, Lab. Chip 2013, 13, 4765.

[61] H. An, H. B. Eral, L. Chen, M. B. Chen, P. Doyle, Soft Matter 2014, DOI 10.1039/C4SM01400F.

[62] D. C. Appleyard, S. C. Chapin, R. L. Srinivas, P. S. Doyle, Nat. Protoc. 2011, 6, 1761. 


\section{Supporting Information}

\section{Biodegradable microparticles for simultaneous detection of counterfeit and deteriorated edible products}

Ivan Rehor, Sophie van Vreeswijk, Tina Vermonden, Wim E. Hennink, Willem K. Kegel, H. Burak Eral*

\section{Synthesis of HEMA- imidazoyl carbamate (HEMA-CI):}

Briefly: 1,1'-carbonyldiimidazole $(2.5 \mathrm{~g}, 15 \mathrm{mmol})$ was placed together with a stirring bar into dry (dried at $150{ }^{\circ} \mathrm{C}$ overnight) $50 \mathrm{ml}$ flask. Apparatus was purged with $\mathrm{N}_{2}$ flow and vacuum (3 times). Then $25 \mathrm{~mL}$ of dry dichloromethane was added. 2-hydroxyethyl methacrylate $(1.0 \mathrm{~g}, 7.7 \mathrm{mmol})$ was added dropwise to the mixture. Reaction was conducted for one hour at RT under $\mathrm{N}_{2}$ atmosphere. Then mixture was extracted with water $(10 \mathrm{~mL}, 1 \mathrm{x})$ and the water extract was washed with dichloromethane $(10 \mathrm{~mL}, 2 \mathrm{x})$. Organic fractions were combined, dried over $\mathrm{MgSO}_{4}$ and $20 \mathrm{mg}$ of 4-methoxyphenol was added to prevent polymerization of the product. Majority of dichloromethane was carefully evaporated on rotavap (15 mins, water bath was kept at RT) to avoid polymerization yielding $1.8 \mathrm{~g}(>100 \%)$ of product in the form of transparent oil. Purity ( ${ }^{1} \mathrm{H}$ NMR): $90 \%$ (residual dichloromethane). $\delta_{\mathrm{H}}\left(400 \mathrm{MHz}, \mathrm{CDCl}_{3}\right) 8.14(\mathrm{~m}, 1 \mathrm{H}, \mathrm{N}-\mathrm{CH}-\mathrm{N}), 7.42(\mathrm{~m}, 1 \mathrm{H}, \mathrm{CO}-\mathrm{N}-\mathrm{CH}-\mathrm{CH}), 7.10(\mathrm{~m}, 1 \mathrm{H}$, CO-N-CH-CH), 6.13 (bs, 1H, C=CH'H'), 5.61 (bs, 1H, C=CH'H') 4.65 (m, 2H, N-CO-O$\left.\mathrm{CH}_{2}\right), 4.50\left(\mathrm{~m}, 2 \mathrm{H}, \mathrm{C}-\mathrm{CO}-\mathrm{O}-\mathrm{CH}_{2}\right), 1.94\left(\mathrm{~m}, 3 \mathrm{H}, \mathrm{CH}_{3}\right)$

\section{Microparticle synthesis and the causes of their nonuniformity:}

The synthetic protocol allows producing particles with sharp features inside the microfluidic channel (Figure S1 left). However the crosslinking density of such hydrogel was relatively 
low, making particles squishy and easily deforming in solution. The low crosslinking density also resulted in their very short shelf life - when stored at RT, they dissolved within several days due to hydrolysis. In order to prelongate their shelf life and to make them mechanically more robust, we increased their crosslinking density after SFL synthesis. After particles were flushed from the microfluidic channel, they were collected in solution containing riboflavin and arginine. The solution with particles was then placed under UV lamp for $20 \mathrm{~min}$. Microphotography of these UV posttreated particles is in Figure 1, 2A, 5 and S1 (right). The features of posttreated particles became better resolved compared to non-posttreated EXP-1 samples (Figure S1 left). Also the shelflife increased to 3 months at RT (see below.). The microphotography of the EXP-2 particles (Figure 2A,S1) show, that not all particles are identical after posttreatment. There is variation in both their size and fluorescence intensity up to $20 \%$. As we did not observe this variation with EXP-1 particles, we conclude, that it comes from their uneven irradiation during posttreatment, caused by their different orientation with respect to the light source. This creates variability in crosslinking density among particles and therefore in particle size (highly crosslinked hydrogel swells less in water solution due to bigger strain). This particle nonuniformity is also main factor responsible for variation in fluorescence decay and particle swelling rates in hydrolysis experiments (see below). We are currently working on procedures which secure that each particle obtains identical light dose in postprocessing step. 


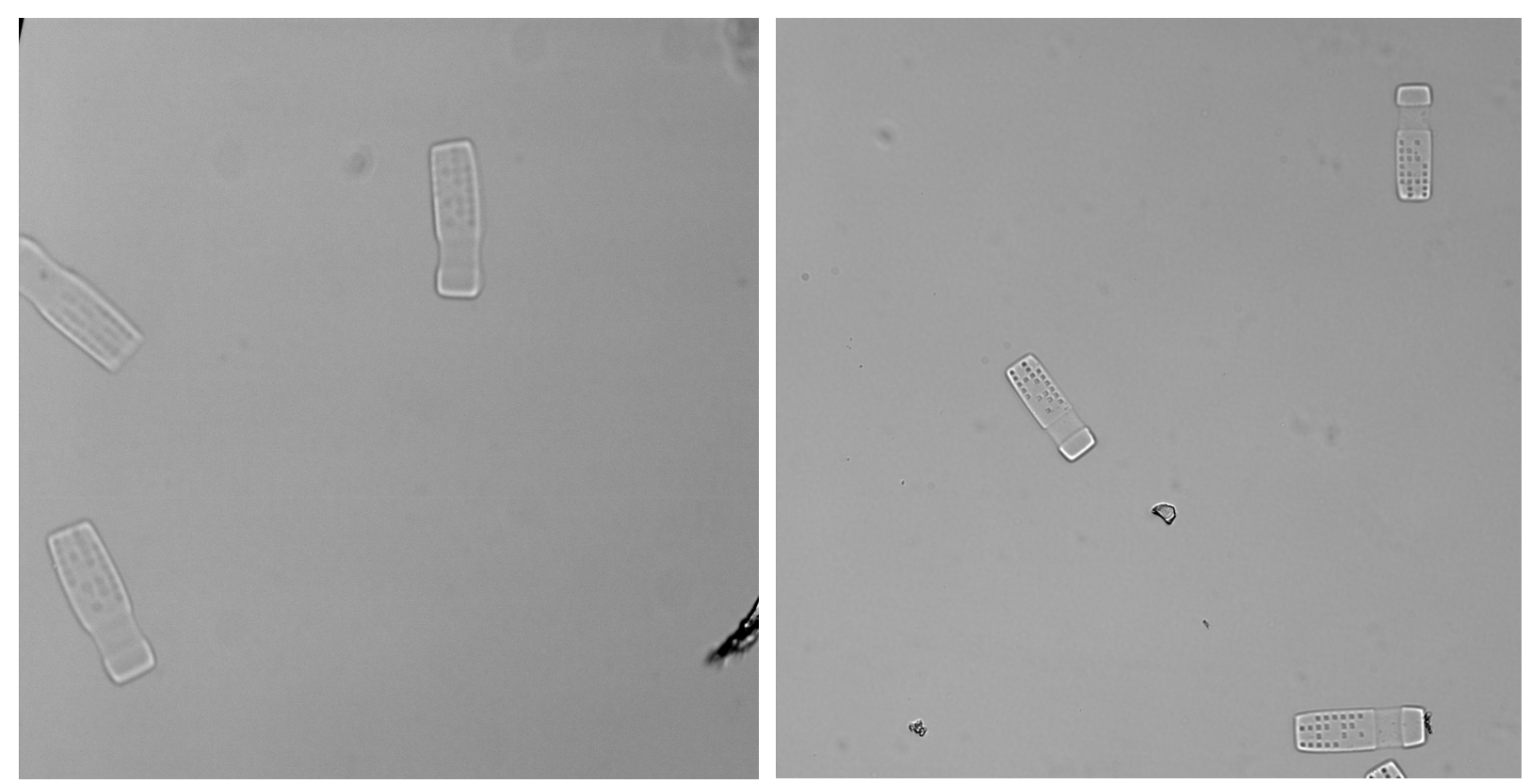

Figure S1. Micrographs of barcode particles EXP-1 (left) and EXP-2 (right). Scale bar corresponds to $100 \mu \mathrm{m}$

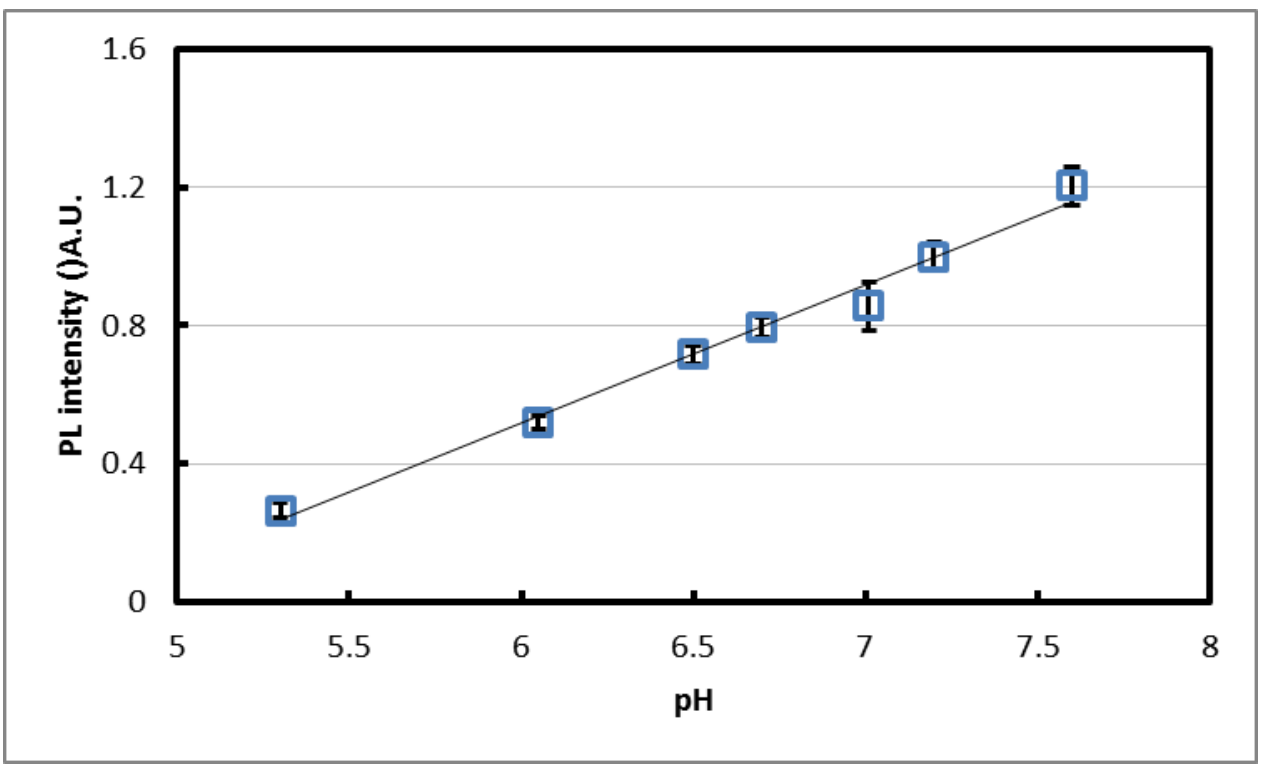

Figure S2. Plot of Fc/Rh emission intensities dependency on $\mathrm{pH}$.

Monitoring of a product storage temperature through the barcode fluorescence decay

The barcode particle is labelled with a methacrylated fluorescent dye, covalently attached to the oligo-HEMA crosslinks. The gradual hydrolysis of Dex-HEMA is therefore accompanied by releasing of the dyed oligo-HEMA crosslings from the hydrogel, which is observed as a decay of fluorescent intensity of a dye $\mathrm{e}^{31}$ (Figure S3). The fluorescent intensity decay plots of 
the particles stored at various temperatures are in in Figure 4B. The plots show decay of the emission intensity to the point, where entire particle completely dissolves. The particle decomposition rate rises with storage temperature. The EXP-1 particles (crosslinked only inside channel - no UV posttreatment) decompose fully in 6 weeks at $4{ }^{\circ} \mathrm{C}$, while at $37^{\circ} \mathrm{C}$ they decompose in only 9 hours. Therefore the level of fluorescence of a barcode dispersed in the liquid product of interest (i.v. solution, oral solution...) give evidence about its storage temperature history. Suggested way of examination is setting a threshold of fluorescence intensity for particular product, below which product is not suitable for use any more. The particle decomposition rate can be tuned. The postproduction irradiation of a particle increases its crosslinking density, which results in a slower decomposition rate. Thus the posttreated particles EXP-2 hydrolyze roughly 20 times slower then EXP-1, for example at $37^{\circ} \mathrm{C}$ it takes them 9 days to dissolve, while EXP-1 dissolves just after 9 hours (Figure S3 and S4). At $4{ }^{\circ} \mathrm{C}$ EXP-2 barcodes remain in solution for $>6$ months. We predict, that varying the postproduction irradiation time will allow fine tuning of the decomposition rate in order to fit to deterioration timescale of a product of interest. An alternative way for tuning the barcode decomposition rate is variation of Dex-HEMA concentration in the pregel solution (Figure S5) or the degree of HEMA substitution ${ }^{34}$. 


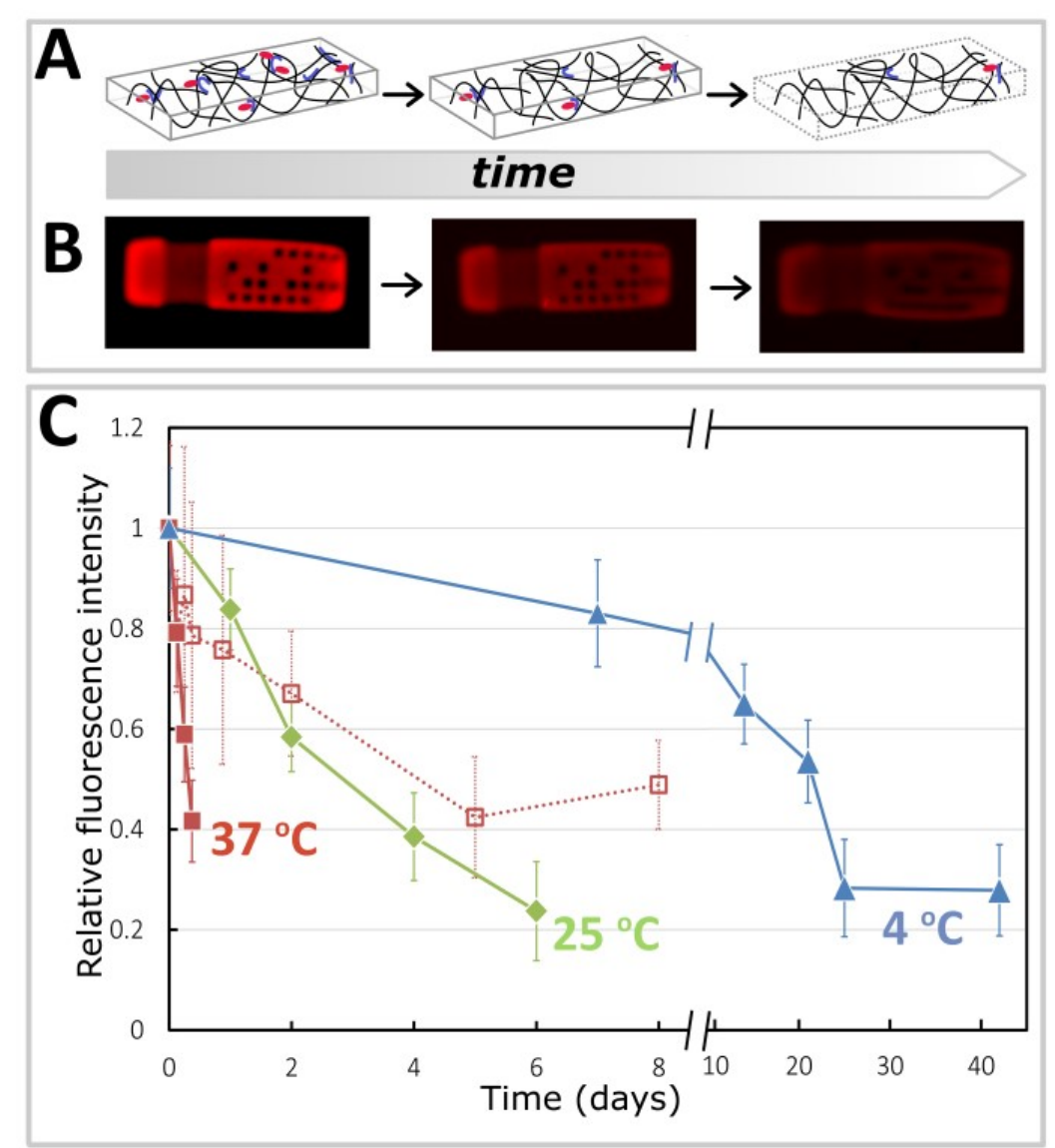

Figure S3. Hydrolysis of barcodes in solution is accompanied by decay of their fluorescence intensity and isused for tracking storage temperature of liquid pharmaceutical product. A The hydrogel consists from Dex-HEMA chains (black) crosslinked with oligoHEMA (blue), which re labelled with a fluorescent dye (red). As carbonate esthers between HEMA and dextran hydrolyze, oligoHEMA chains are washed away from the particle together with the dye. At certain point majority of crosslinks hydrolyses and the barcode dissolves. The hydrolysis is therefore accompanied by gradual decay in dye emission intensity of barcode. B - Micrographs from fluorescent microscope showing gradual decay emission intensity of a barcode in time. $\mathbf{C}$ - Plot of barcode fluorescence over times at various temperatures shows, that temperature significantly affects the hydrolysis rate. One time UV irradiated barcodes EXP-1 fully hydrolyze in 9 hours at $37^{\circ} \mathrm{C}$ (full squares), in 6 days at $25^{\circ} \mathrm{C}$ (diamonds) and in 40 days at $4{ }^{\circ} \mathrm{C}$ (triangles). The difference in hydrolysis rate, which is detected as decay of barcode fluorescence, can therefore be used for tracking storage temperature of liquid pharmaceutical product. The hydrolysis rate is dependent on synthetic conditions of the barcode (exposure, pregel composition...) and can be tuned to correspond to expected shelflife of product of interest. For example second UV irradiation in sample EXP-2 prelongates the lifetime of particles roughly 20 times to 9 days at $37^{\circ} \mathrm{C}$ (dotted line, empty squares). 


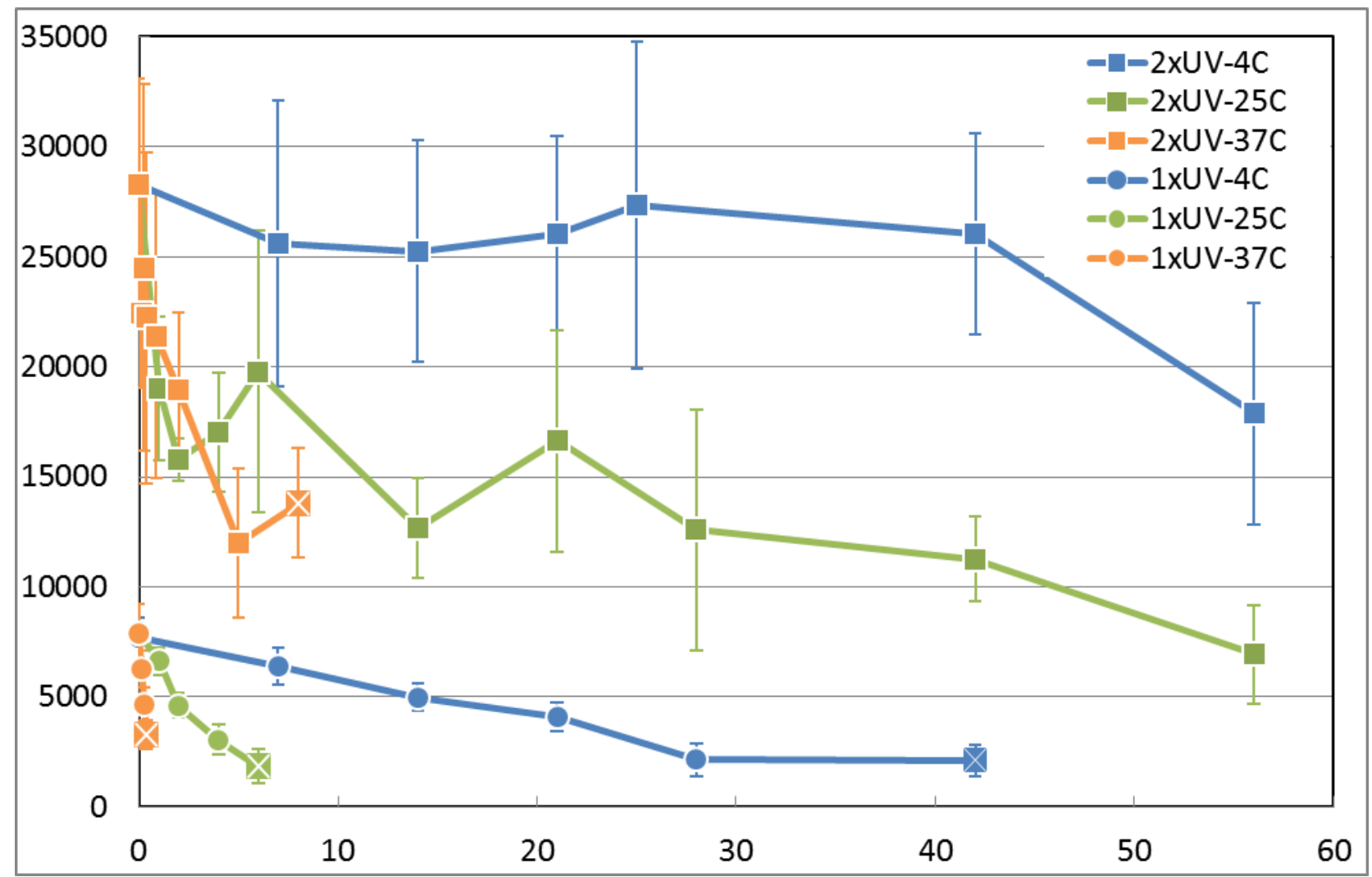

Figure S4. Fluorescence decay of hydrolyzing EXP-1 and EXP-2 particles (full data)

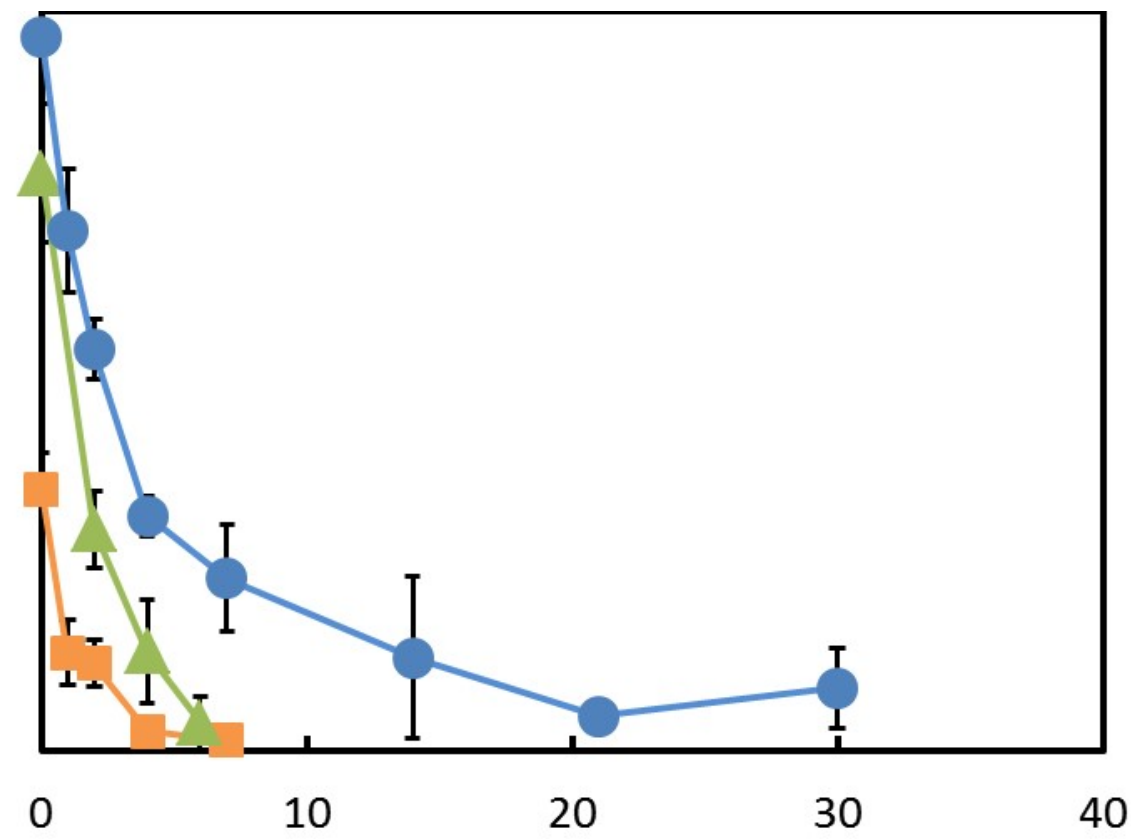

Figure S5. Fluorescence decay of hydrolyzing barcodes, prepared from solutions containing 20 and $25 \%$ (w/w) Dex-HEMA (green and blue respectively). Orange line belongs to smple containing $25 \%$ Dex-HEMA, which was exposure for $1 / 2$ time inside channel. None of the samples was UV posttreated. 


\section{Details of home built SFL setup}

The details of the SFL setup were described previously ${ }^{[42,61,62]}$. Compressed air was used to generate a pressure difference to induce flow in the channel. The input pressure was set to 0.5 psig using a pressure gauge. The gauge outlet was connected to a 3 way solenoid valve which allows switching rapidly between atmospheric pressure (stop) and the input pressure (flow). The solenoid valve was controlled remotely. An inverted microscope was used for particle production. The PDMS channel was placed on the microscope stage. The $0.8 \mathrm{~mm}$ needle with flat end was filled with the pre-gel solution $(50 \mu \mathrm{L}$, standard composition - see above), inserted into the inlet hole and attached to the solenoid valve with a tube. A flat-end needle was inserted to the outlet hole and served for the collection of crosslinked particles. The solenoid valve and the $\mathrm{Hg}$ lamp shutter were programmed to cycle in a following way: I. PURGE: valve opened, shutter closed -3 seconds; II. STOP: valve closed, shutter closed - 1 second; III. PRINT: valve closed, shutter open -1.2

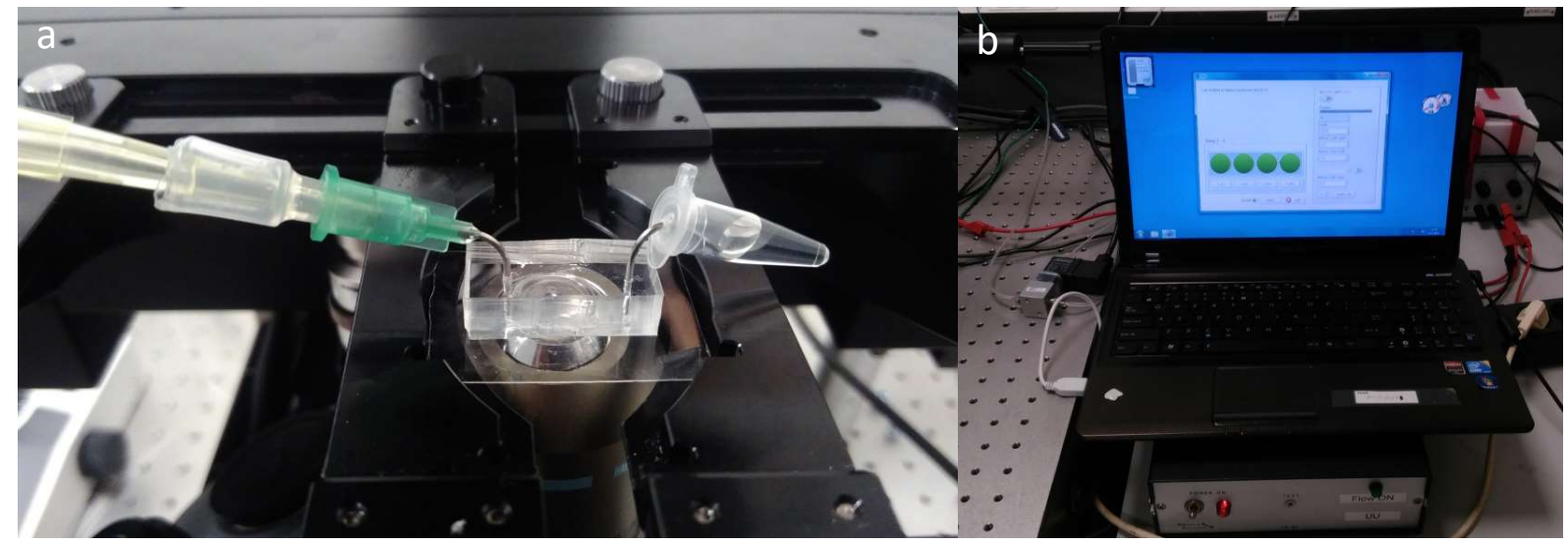

Figure S6 Photos of SFL setup that show the microfluidic setup for SFL and the control circuit consisting of custom written SFL software controlling the shutter and the microfluidic pump 